\title{
Évaluation par cœlioscopie des corps jaunes et des embryons. Influence sur la taille de portée chez la lapine
}

\author{
MA Santacreu *, P Viudes de Castro, A Blasco \\ Universidad Politécnica de Valencia, Dpto Ciencia Animal, \\ Camino de Vera 14, 46020 Valence, Espagne
}

(Reçu le 30 janvier 1990; accepté le 21 juin 1990)

\begin{abstract}
Résumé - Vingt et une lapines ont été l'objet, à 12 j de gestation, d'une évaluation par ccelioscopie de leur taux d'ovulation et du nombre de leurs embryons. Elles ont été aussitôt abattues pour compter les corps jaunes sur l'ovaire et les embryons présents dans l'utérus. La précision de l'évaluation par coelioscopie est très élevée, 91 et $98 \%$ d'exactitude respectivement pour le nombre d'ovulations et celui des embryons. L'effet de la cœlioscopie sur la survie embryonnaire a été observé chez 40 lapines opérées à 12 j de gestation. La cœlioscopie n'a pas d'effet significatif sur la taille de la portée à la naissance, ni sur la gestation en cours ni sur la suivante.
\end{abstract}

coelioscopie / taux d'ovulation / embryon / taille de portée / lapin

Summary - Evaluation by coelioscopy of ovulation rate and embryo number in the rabbit and Its effect on litter slze. Twenty-one does were laparoscopized on d 12 of gestation and immediately killed after observation in order to estimate the ovulation rate and number of embryos. The accuracy was very high: $91 \%$ and $98 \%$ respectively, for ovulation rate and number of embryos. To measure the effect of coelioscopy on fetal survival rate 40 does were laparoscopized on 12 of gestation. The ccelioscopy did not show a significant effect on litter size and birth, or on the current or the subsequent gestation.

coelloscopy / ovulation rate / embryo / litter size / rabbit

\section{INTRODUCTION}

L'amélioration génétique de la taille de portée peut être abordée à travers ses composantes principales, le taux d'ovulation, estimé par le nombre de corps jaunes, et la survie des embryons jusqu'à la mise bas. Classiquement, ces deux paramètres sont mesurés chez la lapine par abattage en cours de gestation ou par laparotomie (Garcia et al, 1983; Torres et al, 1987; Bolet et al, 1988).

\footnotetext{
- Correspondance et tirés à part.
} 
Par rapport à ces deux méthodes, la coelioscopie présente de grands avantages : traumatisme chirurgical et risques sanitaires réduits, et surtout maintien de la vie reproductive de la femelle. II convient cependant de vérifier la fiabilité de cette méthode de comptage des corps jaunes et des embryons, et son innocuité pour la suite de la gestation (viabilité fœtale) et la fertilité ultérieure des lapines.

Theau-Clément et Bolet (1987) ont déjà étudié l'efficacité d'une technique de cœlioscopie pour compter les corps jaunes $8 \mathrm{j}$ après la saillie chez la lapine. L'équipe de Garcia (Molina, 1987) a mis au point une technique permettant de compter à la fois les corps jaunes et les embryons à $12 \mathrm{j}$ de gestation; nous étudions dans cet article sa fiabilité et son influence éventuelle sur la taille de la portée à la naissance.

\section{MATÉRIEL ET MÉTHODES}

\section{Experience 1 : précision de la techni- que de coelioscopie}

\section{Matériel animal}

Vingt et une lapines multipares appartenant aux trois souches de l'élevage expérimental du Département de science animale de I'ETSIA (Valencia, Espagne) ont été utilisées. La souche A est d'origine néo-zélandaise, la souche $R$ d'origine californienne et la souche $V$ est une souche synthétique qui provient de deux hybrides commerciaux, avec une composante importante de race californienne.

Toutes les lapines ont subi une coelioscopie 12 j après la saillie pour compter le nombre de corps jaunes (CL) sur chaque ovaire et le nombre d'embryons vivants (EV) ou en régression $(E R)$ dans chaque corne utérine. Ont été qualifiés d'embryons en régression ceux qui, au moment de l'observation, avaient de visu un diamètre et une vascularisation nettement inférieurs aux autres. Pour chaque femelle, deux observateurs ont complé de manière indépendante les corps jaunes de chaque ovaire et les embryons de chaque corne utérine. Le temps moyen par observateur a été de $8 \mathrm{~min}$. La fomelle a ensuite été abattue pour vérifier le nombre de corps jaunes après énucléation de l'ovaire et le nombre d'embryons vivants ou en régression par comptage direct des embryons.

\section{Technique de cclioscopie}

La technique décrite par Molina (1987) a été utiliséo.

L'anesthésie a élé obtenue avec un mélange 5/1 (V/V) de chlorhydrate de ketamine (ketolar $50 \mathrm{mg} / \mathrm{ml}, 10 \mathrm{ml}$ ) et de prométazine (Phénergan, Rhône-Poulenc, $2 \mathrm{ml}$ ). Une préanesthésie est d'abord pratiquée par injection intramusculaire du mélange, $1,2 \mathrm{ml} / \mathrm{kg}$ de poids vif, puis l'anesthésie proprement dite est obtenue en perfusant la veine marginale de l'oreille avec $1,5 \mathrm{ml}$ du même mélange.

La lapine est ensuite placée sur une table d'opération inclinée vers l'avant de 30 degrés environ. Une aiguille de Veress est introduite sur le côté droit en dessous des dernières côtes pour insuffler du gaz carbonique dans la cavité générale. Une incision médiane à $1 \mathrm{~cm}$ en arrière du sternum permet l'introduction du trocart qui est ensuite retiré du fourreau et remplacé par l'endoscope relié à un générateur de lumière de $250 \mathrm{~W}$. Une autre incision sur le côté droit, 3 $\mathrm{cm}$ en arrière de la précédente, permet l'introduction d'un deuxième trocart qui est ensuite retiré du fourreau et remplacé par une sonde de palpation dont l'extrémité arrondie permet les manipulations nécessaires pour bien mettre en évidence les ovaires et les embryons.

\section{Analyse statistique}

Les données ont été analysées avec le modèle d'analyse de variance suivant :

$$
Y_{i j}=m+O b s_{j}+b x_{i j}+\theta_{i j}
$$

$Y_{i j}$ : variable observée à l'abattage $(\mathrm{CL}, \mathrm{EV}$, $E T=E V+E R$ ).

Obs: effet observateur $(i=1,2)$.

$X_{1 /}$ : nombre observé à la cœlioscopie, correspondant au complage $Y_{i j}$ réalisé à l'abattage. 
$b$ : coefficient de régression de $y$ en $x$.

e : résiduelle.

\section{Expérience 2 : effet de la calioscopie sur la taille de la portée et la vie reproductive ultérieure de la lapine}

\section{Matériel animal}

Cent vingt lapines en deuxième gestation ont été utilisées, appartenant à une souche du même élevage, sélectionnée sur la taille de portée au sevrage : 80 lapines constituaient le groupe témoin (lot $T$ ), 40 autres lapines (lot $L$ ) ont subi une cœlioscopie dans les mêmes conditions que pour l'expérience 1, 12 j après la saillie pour mesurer les mêmes caractères. A la naissance des portées, les nombres de lapereaux nés totaux (NT) ot nés vivants (NV) ont été enregistrés. Quatre-vingt-treize femelles sur 120 (32 dans le lot $L$ et 61 dans le lot $T$ ) ont ensuite produit une troisième portée sur laquelle les nouveau-nés (NT et NY ont été dénombrés.

\section{Analyse statistique}

Les moyennes de $N V$ et $N T$ des deux lots à la deuxième et à la troisième mise bas ont été comparées par un test $t$ de student pour chaque série de naissance.

\section{RÉSULTATS}

\section{Expérience 1}

L'analyse de variance ne montre pas d'effet significatif $(P>0,05)$ de l'observateur sur l'ensemble des 42 ovaires et cornes utérines examinés. Le coefficient de régression entre la mesure par cœlioscopie et celle faite à l'abattage est très proche de l'unité pour les trois caractères étudiés (tableau I). Ceci prouve que la précision du comptage est très élevée -t ne dépend pas du taux d'ovulation : la pı )portion de comptages exacts est de $91 \%$ pour CL et $98 \%$ pour ED et ET. Dans tous les autres cas, la différence entre les deux comptages n'excède jamais \pm 1 ; elle est considérée comme acceptable.

\section{Expérience 2}

II n'y a pas de différences significatives $(P>0,05)$ pour $N V$ et $N T$ entre les deux lots, ni a la mise bas suivant la coelioscopie, ni à la mise bas ultérieure (tableau II).

Tableau I. Résultats des comptages enregistrés à l'abattage. Relation avec ceux observés lors de la coelioscopie.

\begin{tabular}{lccccccc}
\hline & $m$ & $c V$ & $b$ & (SE) & $A^{2}$ & SRD & Sig Obs \\
\hline CL/ovaire & 6,50 & 0,41 & 0,99 & $(0,011)$ & 0,988 & 0,29 & NS \\
EV/corne & 5,43 & 0,45 & 1,10 & $(0,005)$ & 0,998 & 0,10 & NS \\
ET/corne & 5,71 & 0,41 & 1,01 & $(0,005)$ & 0,998 & 0,10 & NS \\
\hline
\end{tabular}

$m$ : moyenne; $C V$ : coefficient de variation; (SE) : écart type de b; $R^{2}$ : coefficient de détermination; SRD : ecart type résiduel; Sig Obs : signification de l'effet observateur; NS : non significatif. 
Tableau Il. Effet de la cœlioscopie sur la gestation de la lapine. La cœelioscopie est pratiquée au $12^{\circ} \mathbf{j}$ de la $2^{\theta}$ gestation sur les lapines du lot $L$.

\begin{tabular}{|c|c|c|c|}
\hline & Lot $L$ & Lot $T$ & Sig \\
\hline \multicolumn{4}{|l|}{$2^{\bullet}$ gestation } \\
\hline Lapines (n) & 40 & 80 & \\
\hline Corps jaunes & $15,2 \pm 2,32^{(1)}$ & - & \\
\hline Embryons vivants & $13,6 \pm 2,75$ & - & \\
\hline Embryons totaux & $13,7 \pm 2,76$ & - & \\
\hline Nés vivants & $9,9 \pm 3,14$ & $10,0 \pm 3,14$ & NS \\
\hline Nés totaux & $10,6 \pm 2,38$ & $10,6 \pm 2,97$ & NS \\
\hline \multicolumn{4}{|l|}{$3^{\bullet}$ gestation } \\
\hline Lapines (n) & 32 & 61 & \\
\hline Nés vivants & $9,9 \pm 3,44$ & $10,4 \pm 3,15$ & NS \\
\hline Nés totaux & $10,5 \pm 3,48$ & $11,6 \pm 2,83$ & NS \\
\hline
\end{tabular}

(1) : moyenne \pm écart type de la moyenne.

Le pourcentage de lapines qui réalisent une troisième mise bas est de $77,5 \%$ ( $80 \%$ du lot $L$ et $76,25 \%$ du lot $T)$. Les éliminations de lapines qui ont dû être faites l'ont toujours été pour des raisons sanitaires et non à cause de difficultés de reproduction.

\section{DISCUSSION}

La précision du comptage des corps jaunes par ccelioscopie rapportée dans ce travail est supérieure à celle obtenue dans les travaux déjà publiés sur ce sujet.

Chez la lapine au $8^{\theta} \mathrm{j}$ de gestation, Theau-Clément et Bolet (1987) ont obtenu des résultats moins précis pour le nombre de corps jaunes, $45 \%$ et $80 \%$ respectivement de comptages exacts et acceptables. Chez le porc, la précision est encore inférieure, $27 \%$ et $70 \%$ de comptages exacts et acceptables (Locatelli,1971). Les diffé- rences de précision dans le comptage des corps jaunes peuvent être dues aux différences de technique employée etou à l'expérience de l'observateur. Theau-Clément et Bolet (1987) introduisaient l'endoscope en arrière de l'attache ombilicale. Les deux endoscopes employés avaient un champ de vision de 70 degrés, 12 et $15 \mathrm{~cm}$ de longueur et un diamètre de 3,5 et $4,5 \mathrm{~cm}$. Dans notre cas, l'endoscope a un champ de 30 degrés, $40 \mathrm{~cm}$ de longueur et $6,5 \mathrm{~cm}$ de diamètre et permet d'avoir un champ de vision plus grand. Le lieu d'introduction et la longueur de l'endoscope permettent d'avoir une meilleure vision de l'ensemble du tractus génital. Ceci facilite le comptage des embryons et donne une vision des deux faces des ovaires. L'alignement de l'endoscope, de la sonde de palpation et du hile ovarien facilite le comptage des corps jaunes. Ceux qui se trouvent sous le pavillon sont les plus difficiles à observer. Certaines erreurs peuvent être dues à la grande proximité de certains corps jaunes 
qui sont alors comptés comme un seul. D'autres résultent de la ressemblance de certaines structures, comme les follicules lutéinisés, avec des corps jaunes. Nous n'avons trouvé dans la bibliographie aucune référence concernant la précision du comptage des embryons par laparoscopie.

Nous n'avons observé aucun effet de la coelioscopie sur la suite de la gestation, ni sur la reproduction ultérieure des lapines (acceptation du mâle, fertilité, prolificité) confirmant ainsi les travaux antérieurs (Wildt et al, 1975; Oldham et al, 1976; Seeger, Klatt, 1980; Wildt, 1980; Harrison, 1980; Dukelow, 1980).

Chez la lapine, cette technique est très utile dans les études sur l'ovulation (Fujimoto et al, 1974), la mortalité embryonnaire (Molina, 1987) ou de la mise en place des embryons dans les cornes utérines (Santacreu et al, 1990).

II faut néanmoins souligner la nécessité de réaliser l'opération et la manipulation des organes avec le plus grand soin, afin de ne pas provoquer d'effets ultérieurs indésirables, notamment en raison de la formation d'adhérences au niveau de l'oviducte ou de l'utérus.

\section{CONCLUSION}

Nos résultats permettent donc de conclure que, par sa précision et l'absence d'effets ultérieurs défavorables, la technique de cœlioscopie que nous avons décrite peut se substituer aux techniques utilisées antérieurement chez la lapine pour compter les corps jaunes et les embryons.

\section{REMERCIEMENTS}

Les auteurs expriment leur gratitude à F Garcia Ximénez pour les avoir initiés à cette technique de cœlioscopie et pour son aide précieuse lors de la réalisation de cette étude.

\section{RÉFÉRENCES}

Bolet G, Brun JM, Hulot F (1988) Relation between ovulation rate and embryonic survival in various strains of rabbits. IVth World Rabbit Congr, 10-14 octobre 1988, Budapest, 149-157

Dukelow WR (1980) Laparoscopy in small animals and ancillary techniques. In: Animal Laparoscopy (Harrison RM, Wildt DE, eds) Williams \& Wilkins Company, Baltimore

Fujimoto S, Rawson JMR, Dukelow WR (1974) Hormonal influences on the time of ovulation in the rabbit as determined by laparoscopy. J Reprod Fertil 38, 97-103

Garcia F, Baselga M, Pla M (1983) Mortalidades embrionaria y fetal en las distintas etapas de la gestación en el conejo de carne. An Inst Nac Invest Agrar Ser Ganad 18, 11-27

Harrison RH (1980) Laparoscopy in monkeys and apes. In: Animal Laparoscopy (Harrison RM, Wildt DE, eds) Williams \& Wilkins Company, Baltimore, 73-93

Locatelli A (1971) Technique d'examen cœelioscopique des ovaires de la truie. Ann Biol Anim Biochim Biophys 11, 495-498

Molina I (1987) Determinación de los efectos genéticos directos y maternos sobre la tasa de ovulacion y la viabilidad embrionaria y fetal en el conejo, mediante aplicación de las técnicas de transferencia de embriones y laparoscopía. Thèse de doctorat, Univ Polytech Valence (Espagne)

Oldham CM, Knight TW, Lindsay DR (1976) A comparison of the effects on reproductive performance in sheep of two methods of estimation of ovulation rate. Austr J Exp Agric Anim Husb 16, 24-27

Santacreu MA, Vicente J, Garcia F (1990) Embryos transfer by laparoscopy in rabbit. $5^{\text {es }}$ Journées de la Rech Cunicole, Paris (sous presse)

Seeger KH, Klatt PR (1980) Laparoscopy in the sheep and goat. In: Animal Laparoscopy (Harrison RM, Wildt DE, eds) Williams \& Wilkins Company, Baltimore, 107-120 
Theau-Clément M, Bolet G (1987) Mesure du taux d'ovulation par coelioscopie chez la lapine. Reprod Nutr Dev 27, 701-705

Torres S, Hulot F, Meunier M, Sevellec C (1987) Comparative study of preimplantation development and embryonic loss in two rabbit strains. Reprod Nutr Dev 27, 707-714
Wildt DE, Morcon CB, Dukelow WR (1975) Laparoscopic pregnancy diagnosis and uterine fluid recovery in swine. J Reprod Fertil 44, 301-304

Wildt DE (1980) Laparoscopy in the dog and cat. In: Animal Laparoscopy (Harrison RM, Wildt DE, eds) Williams \& Wilkins Company, Baltimore, 31-72 et 121-132 